\title{
Canagliflozin-induced pancreatitis: a rare side effect of a new drug
}

\author{
This article was published in the following Dove Press journal: \\ Therapeutics and Clinical Risk Management \\ 26 June 2015 \\ Number of times this article has been viewed
}

\author{
Mudit Chowdhary' \\ Ahmad A Kabbani' \\ Akansha Chhabra ${ }^{2}$ \\ 'Department of Internal Medicine, \\ Mercer University School of Medicine, \\ Macon, GA, USA; ${ }^{2}$ Department \\ of Internal Medicine, New York \\ University Langone Medical Center, \\ New York, NY, USA
}

\begin{abstract}
Acute pancreatitis is most commonly attributed to gallstones, alcohol abuse, and metabolic disorders such as hyperlipidemia and hypercalcemia. Medications are an infrequent yet commonly overlooked etiology of pancreatitis. Although several drugs have been implicated, antidiabetic agents are a rare cause for drug-induced pancreatitis. Canagliflozin is a new drug in the class of SGLT-2 inhibitors used for the treatment of type 2 diabetes mellitus. Serious reported side effects include renal impairment, hyperkalemia, and hypotension. Pancreatitis as a result of canagliflozin, however, is exceedingly rare. Here we describe a case of a 33-year old female who presented with severe acute pancreatitis in the setting of recent initiation of canagliflozin. Given the timing of her presentation and after excluding all other possible etiologies, it was determined that canagliflozin was the likely source of her illness. This case highlights the importance of identifying drug-induced pancreatitis, especially in novel drugs, as it is commonly neglected in patients with multiple medical comorbidities and those taking numerous medications. Prompt identification of drug-induced pancreatitis can improve management as well as decrease morbidity and mortality in these individuals.
\end{abstract}

Keywords: canagliflozin, Invokana, pancreatitis, drug-induced pancreatitis, SGLT-2 inhibitor

\section{Introduction}

Acute pancreatitis (AP) is an inflammatory condition of the pancreas characterized clinically by intense epigastric pain radiating to the back along with elevated levels of pancreatic enzymes in the blood. Although pancreatitis is a leading cause of hospitalization in the United States, ${ }^{1}$ the pathogenesis for this condition is not fully understood. Nevertheless, various etiologies have been shown to increase the risk for and to cause pancreatitis.

Gallstones are the most common cause for AP, accounting for 35\%-40\% of cases worldwide, and together with alcohol, and metabolic disorders such as hyperlipidemia and hypercalcemia make up around $90 \%$ of all cases. ${ }^{2}$ Medications are infrequently associated with pancreatitis with a reported incidence of only $0.1 \%-2 \%{ }^{3,4}$ although several drugs have been implicated including diuretics, didanosine, tetracycline, sulfonamides, and steroids, among others.

Diabetic medications have rarely been associated with pancreatitis with the majority of reported cases involving DPP-4 inhibitors (sitagliptin, saxagliptin) and GLP-1 receptor antagonists (exenatide, liraglutide). ${ }^{5}$ Canagliflozin is a new drug in the class of sodium-glucose cotransporter-2 (SGLT-2) inhibitors used for the treatment of type 2 diabetes mellitus (T2DM). Here we describe a patient who developed a severe manifestation of AP soon after initiation of canagliflozin therapy.
Correspondence: Mudit Chowdhary Department of Internal Medicine, Mercer University School of Medicine, 707 Pine Street, Macon, GA 3I20I, USA

Email mu.chowdhary@gmail.com BY LC License. The full terms of the License are available at http://creativecommons.org/licenses/by-nc/3.0/. Non-commercial uses of the work are permitted without any further (c) (i) (5) 2015 Chowdhary et al. This work is published by Dove Medical Press Limited, and licensed under Creative Commons Attribution - Non Commercial (unported, v3.0) permission from Dove Medical Press Limited, provided the work is properly attributed. Permissions beyond the scope of the License are administered by Dove Medical Press Limited. Information on
how to request permission may be found at: http://www.dovepress.com/permissions.php 


\section{Case presentation}

A 33-year old African-American female presented to the emergency room with a 2-day history of progressively worsening nausea, vomiting, and severe abdominal pain. The patient denied any cardiovascular, respiratory, or urinary symptoms nor had she been around any sick contacts. The patient denied any abdominal surgeries or prior history of gallstones, dyslipidemia or pancreatitis. She denied any alcohol, cigarette use, or illicit drug use. She had a history of diabetes mellitus, hypertension and hypothyroidism; however, there was no family history of autoimmune conditions or AP. Her home medications included long-term metformin and levothyroxine. In addition, the patient recently began canagliflozin therapy 2 weeks prior to her admission.

In the emergency department, she was found to be hypotensive with a blood pressure of 79/36, heart rate of $118 \mathrm{bpm}$, and a respiratory rate of 27 . Her temperature was 40.3 degrees Celsius. Serum chemistries revealed leukocytosis with a white blood cell count of $23.6 \times 10^{3} / \mathrm{mm}^{3}$, creatinine of $3.19 \mathrm{mg} / \mathrm{dL}$, calcium of $9.3 \mathrm{mg} / \mathrm{dL}$, amylase $535 \mathrm{IU} / \mathrm{L}$, and lipase $373 \mathrm{IU} / \mathrm{L}$. $\mathrm{HbA}_{1 \mathrm{c}}$ was recorded as $13.5 \%$ and her triglyceride level was within normal limits. Additionally, she displayed an elevated anion gap of 19 and acidosis with a $\mathrm{pH}$ of 6.89 with blood glucose level of $563 \mathrm{mg} / \mathrm{dL}$ and positive urine ketones (beta-hydroxybutyrate: 2.90 ). Subsequent arterial blood gas revealed PCO2 of 48.8 and FiO2 of 100. Maximum blood alcohol was negative. She was initially diagnosed with diabetic ketoacidosis (DKA) and given several liters of normal saline followed by a bicarbonate and insulin drip. Urine and blood cultures were drawn and she was started on broad-spectrum antibiotics for her fever and leukocytosis. A computerized tomography (CT) scan of the abdomen was obtained due to the elevated pancreatic enzymes and it showed evidence of AP with peripancreatic inflammation and ascites (Figure 1). Chest X-ray additionally revealed bilateral pleural effusions. Echocardiogram though was unremarkable.

Despite aggressive fluid resuscitation, the patient became progressively more hypotensive and became unresponsive. She was started on a norepinephrine drip, intubated and admitted to the intensive care unit. Her renal function deteriorated with decreased urine output and increasing serum creatinine reaching 4.26 (pre-morbid creatinine: 1.0) with persistent acidemia requiring continuous renal replacement therapy. In the intensive care unit, an APACHE (Acute Physiology and Chronic Health Evaluation) II score was calculated for the patient and revealed an estimated mortality rate of $97.2 \%$.

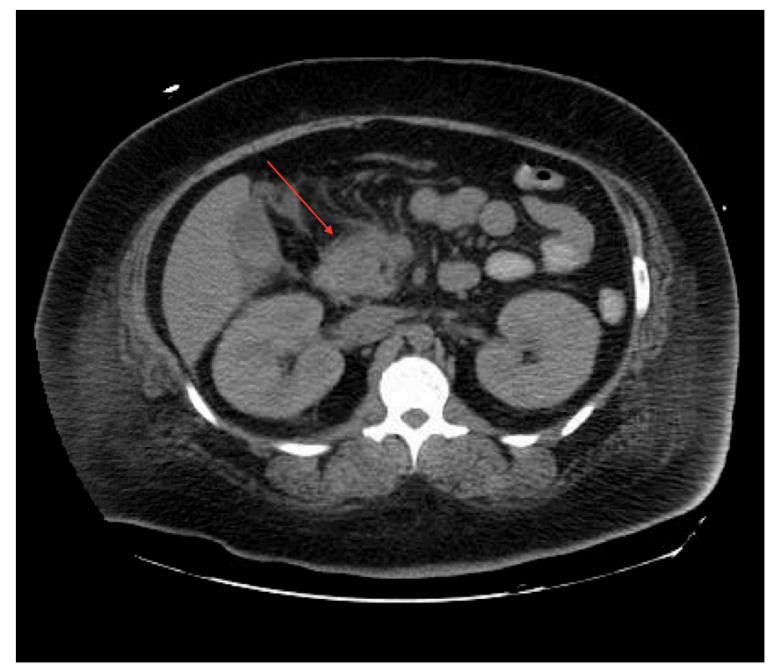

Figure I Abdominal computerized tomography scan revealing acute pancreatitis (red arrow).

Despite the poor prognosis the patient's condition remarkably improved and vasopressors were discontinued and she was successfully extubated after 4 days. The source of leukocytosis was indeterminate as cultures of blood, urine, and mini bronchoalveolar lavage were all negative. After excluding all other causes for her symptoms and given the chronology of her presentation, it was determined that canagliflozin was likely the source of her illness. Canagliflozin was discontinued and the patient has remained stable since discharge.

\section{Discussion}

While metformin has been widely accepted as the initial drug of choice for T2DM ${ }^{6}$ numerous second-line classes of diabetic medications exist for clinicians to choose from, with individual choice determined largely by factors including cost and risk for adverse effects. Canagliflozin is the first SGLT-2 inhibitor approved by the FDA (US Food and Drug Administration) for the treatment of T2DM. ${ }^{7}$

Normally, plasma glucose is freely filtered by the glomerulus and reabsorbed in the proximal renal tubule, with a small amount excreted directly in the urine. Ninety percent of renal glucose reabsorption occurs in the proximal tubule as a result of SGLT-2. ${ }^{8}$ Canagliflozin works by inhibiting SGLT-2, causing a dramatic reduction in the kidneys' capacity to reabsorb glucose, thus leading to a decrease in plasma glucose levels. ${ }^{9}$ Clinical studies have shown remarkable efficacy with this drug. The Canagliflozin Treatment and Trial Analysis-Monotherapy Trial demonstrated a baseline $\mathrm{HbA}_{1 \mathrm{c}}$ reduction by $0.91 \%-1.16 \%$, a reduction in systolic blood pressure and body weight, and an increased high-density 
lipoprotein level. ${ }^{10}$ Additionally, studies have also shown a $0.81 \%-0.93 \%$ decrease in $\mathrm{HbA}_{1 \mathrm{c}}$ when canagliflozin was given in conjunction with metformin. ${ }^{11}$

The most common side effects of canagliflozin are urinary tract infections and genital mycotic infections, which occur more frequently in women. Additional potential side effects include nausea, renal impairment, hyperkalemia, and hypoglycemia. Pancreatitis as a result of canagliflozin is exceedingly rare, with an incidence rate of only 2.7 per 1,000 patient-years. $^{12}$

The exact mechanism of canagliflozin-induced pancreatitis is currently unclear; however, the underlying pathogenesis is unlikely to differ from other causes of AP. In fact, the majority of drug-induced pancreatitis (DIP) appears to be idiosyncratic in nature. ${ }^{13}$ Idiosyncratic drug reactions are adverse effects not directly related to pharmacodynamic drug mechanisms. These spontaneous and unpredictable adverse complications are a result of abnormal interactions between specific drugs and the patient themselves, and are typically mediated by immunologic or cytotoxic effects triggered by the drug or its metabolites on a specific organ system. ${ }^{14}$

Nevertheless, DIP is a serious complication and very easily overlooked in patients with multiple comorbidities, those on multiple medications, or those with potential underlying risk factors such as gallstones or alcohol abuse.

We believe this is the second reported incidence of canagliflozin-induced pancreatitis as a PubMed literature search for several variations of "canagliflozin" and "pancreatitis" revealed only one additional case. ${ }^{15}$ Our case is further unique in that pancreatitis was also associated with acute kidney injury. It remains to be seen whether kidney injury was a sequela of the pancreatitis or a simultaneous additional side effect of canagliflozin. It is important to note that the patient had been on long-term metformin and levothyroxine when this occurred, albeit without a history of pancreatitis or other side effects. Several case reports have suggested a possible role of metformin in causing AP, specifically during periods of renal insufficiency. ${ }^{16,17}$ Therefore, it is possible that metformin also played a role in precipitating this episode of AP.

Special care must also be given to patients who present with DKA and concurrent abdominal pain. Nair et al ${ }^{18}$ reported an association between DKA and AP, though the pathogenesis primarily involved transient hyperlipidemia rather than DIP. Regardless, DKA may mask a coexisting AP in $10 \%-15 \% .{ }^{18}$ The key treatment for DIP is early recognition, exclusion of all other etiologies, and discontinuation of the possible causative medication followed by supportive therapy.
In our case, the adverse event occurred within a reasonable period of drug administration. Laboratory data and radiological imaging confirmed the diagnosis of AP. Additionally, there were no other changes to the patient's drug regimen in the weeks preceding pancreatitis aside from the addition of canagliflozin. Furthermore, the event was not attributable to any other etiology, as our patient did not have a history of recent infection, gallstones, hypertriglyceridemia, or alcohol intake. Finally, there was no recurrence of pancreatitis following discontinuation of canagliflozin. Therefore, according to the Naranjo probability scale, the adverse event was most likely related to canagliflozin. ${ }^{19}$

\section{Conclusion}

As the prevalence of diabetes in the United States increases, the amount of new medications designed to combat it will increase as well. Our case illustrates the importance of being aware of new antidiabetic drugs such as canagliflozin as potential treatment options for T2DM as well as remaining up to date on potential side effects and drug interactions of these newer agents. DIP is an uncommon yet potentially serious complication of canagliflozin. It remains to be seen whether this is as a result of canagliflozin monotherapy or due to an interaction with metformin in the setting of acute kidney injury. Regardless, DIP may easily be overlooked in patients due to its low incidence, and confounded by the presence of comorbidities, and potential of multiple medication use. Thus it is imperative for clinicians to keep a high index of suspicion in order to prevent morbidity and mortality from disease progression.

\section{Acknowledgment}

No outside funding was utilized in this study.

\section{Disclosure}

The authors declare that there is no conflict of interests in this work.

\section{References}

1. Peery AF, Dellon ES, Lund J, et al. Burden of gastrointestinal disease in the United States: 2012 update. Gastroenterology. 2012;143(5):1179-1187. e1-3.

2. Forsmark CE, Baillie J; AGA Institute Clinical Practice and Economics Committee; AGA Institute Governing Board. AGA Institute technical review on acute pancreatitis. Gastroenterology. 2007;132(5): 2022-2044.

3. Spanier BW, Tuynman HA, van der Hulst RW, Dijkgraaf MG, Bruno MJ. Acute Pancreatitis and Concomitant Use of Pancreatitis-Associated Drugs. Am J Gastroenterol. 2011;106(12):2183-2188.

4. Balani AR, Grendell JH. Drug-induced pancreatitis: incidence, management and prevention. Drug Saf. 2008;31(10):823-837. 
5. Cohen D. Reports of pancreatitis are 20-30 times more likely with GLP-1 drugs, analysis finds. BMJ. 2013;346:f2607.

6. American Diabetes Association. Standards of medical care in diabetes - 2014. Diabetes Care. 2014;37 Suppl 1:S14-S80.

7. US Food and Drug Administration. FDA approves Invokana to treat type 2 diabetes [press release]. US Food and Drug Administration; 2013. Available from: http://www.fda.gov/NewsEvents/Newsroom/ PressAnnouncements/ucm345848.htm. Accessed February 22, 2015.

8. Chao EC, Henry RR. SGLT2 inhibition-a novel strategy for diabetes treatment. Nat Rev Drug Discov. 2010;9(7):551-559.

9. Sha S, Devineni D, Ghosh A, et al. Canagliflozin, a novel inhibitor of sodium glucose co-transporter 2, dose dependently reduces calculated renal threshold for glucose excretion and increases urinary glucose excretion in healthy subjects. Diabetes Obes Metab. 2011;13(7):669-672.

10. Stenlof K, Cefalu WT, Kim KA, et al. Efficacy and safety of Canagliflozin monotherapy in subjects with type 2 diabetes mellitus inadequately controlled with diet and exercise. Diabetes Obes Metab. 2013;15(4):372-382.

11. Valentine V, Hinnen D. Clinical implications of canagliflozin treatment in patients with type 2 diabetes. Clin Diabetes. 2015;33(1):5-13.
12. Invokana ${ }^{\circledR}$ (canagliflozin) [prescribing information]. Titusville, NJ: Janssen Pharmaceuticals, Inc.; 2014.

13. Hung WY, Abreu Lanfranco O. Contemporary review of drug-induced pancreatitis: A different perspective. World J Gastrointest Pathophyiol. 2014;5(4):405-415.

14. Badalov N, Baradarian R, Iswara K, Li J, Steinberg W, Tenner S. Drug-induced acute pancreatitis: an evidence-based review. Clin Gastroenterol Hepatol. 2007;5(6):648-651.

15. Verma R. Canagliflozin-Associated Acute Pancreatitis. Am J Ther. Epub 2014 Sep 2.

16. Fimognari FL, Corsonello A, Pastorell R, Antonelli-Incalzi R. Metformin-induced pancreatitis. Diabetes Care. 2006;29(5):1183.

17. Alsubaie S, Almalki MH. Metformin induced acute pancreatitis. Dermatoendocrinol. 2013;5(2):317-318.

18. Nair S, Yadav D, Pitchumoni CS. Association of diabetic ketoacidosis and acute pancreatitis: observations in 100 consecutive episodes of DKA. Am J Gastroenterol. 2000;95(10):2795-2800.

19. Naranjo CA, Busto U, Sellers EM, et al. A method for estimating the probability of adverse drug reactions. Clin Pharmacol Ther. 1981; 30(2):239-245.
Therapeutics and Clinical Risk Management

\section{Publish your work in this journal}

Therapeutics and Clinical Risk Management is an international, peerreviewed journal of clinical therapeutics and risk management, focusing on concise rapid reporting of clinical studies in all therapeutic areas, outcomes, safety, and programs for the effective, safe, and sustained use of medicines. This journal is indexed on PubMed Central, CAS,

\section{Dovepress}

EMBase, Scopus and the Elsevier Bibliographic databases. The manuscript management system is completely online and includes a very quick and fair peer-review system, which is all easy to use. Visit $\mathrm{http}: / /$ www.dovepress.com/testimonials.php to read real quotes from published authors. 